\title{
Steady-State Throughput Analysis of Network Coding Nodes Employing Stop-and-Wait Automatic Repeat Request
}

\author{
Yang Qin, Student Member, IEEE, and Lie-Liang Yang, Senior Member, IEEE
}

\begin{abstract}
This paper analyzes the steady-state throughput of network coding nodes when data is transmitted based on the stopand-wait automatic repeat request (SW-ARQ) scheme. The state transition of network coding nodes employing SW-ARQ is analyzed, which shows that the operations of network coding nodes can be modeled by a finite state machine. Therefore, the throughput expressions of network coding nodes can be derived based on the properties of finite state machines. Furthermore, the throughput performance of network coding nodes is investigated either by simulations or by evaluation of the expressions obtained. It can be shown that the simulation results converge closely to the numerical results and justify the effectiveness of our analytical expressions obtained.
\end{abstract}

Index Terms-Automatic repeat request (ARQ), network coding, network coding node, state machine, throughput.

\section{INTRODUCTION}

$\mathbf{N}$ ETWORK coding has received a lot of attention, since its invention by Ahlswede et al. [1]. Network coding deals with the problems of coding over packet networks, and it has been recognized that the network-coding-assisted routing has the potential to outperform the conventional routing [1], [2]. Performance of communication systems with network coding has been investigated, when assuming that packets are conveyed error-free in the networks [3]. However, in practice, transmission errors always occur, and error-detection or error-correction techniques are often required in order to ensure reliable communications [2], [4]. Therefore, in this paper we motivate to study the steady-state throughput of the network coding nodes, where communications between two nodes are based on the stop-and-wait automatic repeat request (SW-ARQ) data transmission scheme [5]-[7].

Here, the throughput of a network coding node is defined as the rate that coded packets are correctly conveyed by the network coding node to its following node(s). Note that we focus our attention on the steady-state throughput of network coding node since, when communicating over unreliable channels, the

Manuscript received October 16, 2009; revised October 18, 2010 and May 30, 2011; accepted November 15, 2011; approved by IEEE/ACM TRANSACTIONS ON NETWORKING Editor S. Shakkottai. Date of publication December 22, 2011; date of current version October 11, 2012. This work was presented in part at the IEEE Vehicular Technology Conference (VTC2010-Spring), Taipei, Taiwan, May 16-19, 2010.

The authors are with the Faculty of Physical and Applied Science, School of Electronics and Computer Science, University of Southampton, Southampton SO17 1BJ, U.K. (e-mail: yq06r@ecs.soton.ac.uk; 1ly@ecs.soton.ac.uk).

Digital Object Identifier 10.1109/TNET.2011.2178860 achievable throughput of a communication network with network coding nodes is mainly constrained by the throughput of the network coding nodes, as shown in the butterfly network that will be detailed in Section III-A.

More specifically, in this paper, the steady-state throughput of the network coding node is analyzed, where the coding node is associated with multiple-input-single-output (MISO) links connecting the coding node with multiple source nodes and one sink node [8], [9]. Therefore, we refer to it as the MISO network coding node. At our MISO network coding node, we assume that there is a buffer storing at most one packet for each of the incoming and outgoing links, which are supported by the SW-ARQ data transmission scheme. Specifically, we first analyze in detail the operations, properties, and steady-state throughput of a two-input-single-output (2ISO) network coding node supported by the SW-ARQ transmission scheme. It can be shown that the operations of the network coding nodes can be modeled by a state machine working in the principles of discrete-time Markov chain, which is convenient for analyzing the steady-state throughput. Then, the analytical approaches for deriving the steady-state throughput of the 2ISO network coding node is extended to the MISO network coding nodes. Finally, the throughput performance of network coding nodes with different numbers of input links is investigated by both simulation and numerical approaches. Our performance results demonstrate that the simulation results converge well to the numerical results, which justifies the effectiveness of our analytical expressions derived.

It is worthy of mentioning that our analytical approaches proposed and the expressions derived can be applied not only to linear network coding, but also to the other families of network coding schemes, provided that, in these network coding schemes, a network coding node requires one packet from each of the incoming links before encoding a new outgoing packet. Furthermore, our study of the single network coding node may be extended to the networks having multiple network coding nodes. For example, once the throughput values of the network coding nodes in a network are respectively obtained, the achievable throughput of the network, or its approximation, may be found with the aid of the max-flow min-cut principles [10]. However, we should realize that the throughput analysis of the networks with multiple network coding nodes is much more challenging than that of the single network coding node. Hence, the throughput analysis of networks with multiple coding nodes constitutes one of the open problems that require further research. 
The remainder of this contribution is organized as follows. Section II provides a review for the work related to this paper. Section III considers the 2ISO network coding system, where system model, representation of coding node's operations, and steady-state throughput are considered. Then, in Section IV, the steady-state throughput of the MISO network coding node is addressed. Performance results are provided in Section V, and finally, in Section VI, we provide the conclusions.

\section{RELATED WORK}

In this section, we provide a brief summary of the existing references related to our work in this paper. Network coding with feedback has first been considered in [11], where the authors use some simple examples to indicate that using feedback associated with network coding may be beneficial to parameter adaptation, reliability enhancement, and packet acknowledgment in network coding systems. In [12], the authors have introduced buffer into random network coding in order to overcome the problem due to asynchronous arrivals of incoming packets. In [13], an ARQ-assisted network coding scheme, namely the "drop-when-seen" algorithm, has been proposed and studied for the sake of reducing queue size. Motivated to reduce the average decoding delay, this ARQ-assisted network coding scheme has also been extended to the three-receiver case [14], where one transmitter communicates with multiple receivers. To be in a little more detail, in the ARQ-assisted drop-when-seen network coding, the transmitter broadcasts linear combinations of the packets stored in its buffer. In order to decide which packets are combined for transmission, in [13] and [14], the concept of "seen" packets has been introduced, which can be explained as follows. Let us denote a packet by $\boldsymbol{p}$. Then, $\boldsymbol{p}$ is said to be seen by a receiver if it is capable of computing the linear combination of $\boldsymbol{p}+\boldsymbol{q}$, where $\boldsymbol{q}$ is a packet linearly combined only by the packets arriving at this receiver later than the packet $\boldsymbol{p}$. In the ARQ-assisted drop-when-seen network coding, once a packet $\boldsymbol{p}$ is seen by all the receivers concerned, then, the packet $\boldsymbol{p}$ can be removed from the transmitter's buffer. In this manner, the transmission queue can be kept relatively short [13], while the average decoding delay can be reduced [14].

Additionally, there are several schemes, which have been proposed in order to improve the delay performance of the ARQ-assisted network coding schemes. Specifically, in [15], the authors have suggested to transmit uncoded packets at critical moments, so as to meet the delay requirement. By contrast, in [16], the authors have developed a feedback-based adaptive broadcast coding scheme in order to reduce the delay for the applications where packets must be accepted in order. Furthermore, in contrast to two-hop networks, as the one considered in this paper, some network coding schemes have been proposed and analyzed in the context of single-hop networks. For example, in [17], a random network coding framework employing hybrid ARQ scheme has been proposed for single-hop real-time media broadcast. In [10], a queuing-based dynamic network coding scheme has been studied in the context of the single-hop networks consisting of one source node and multiple receivers. The

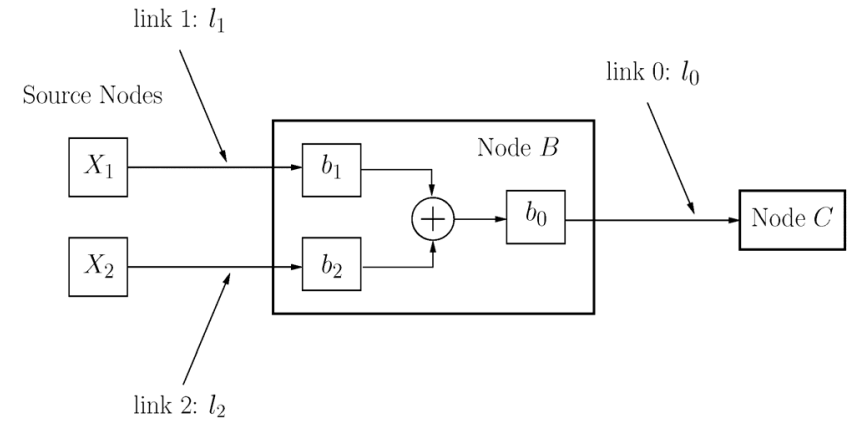

Fig. 1. Network coding node $B$ with two incoming links $l_{1}, l_{2}$ and one outgoing link $l_{0}$.

studies in [10] illustrate that this network coding scheme is capable of asymptotically achieving both the throughput of near the max-flow min-cut bound and the channel erasure probability of near zero.

Against this background, in this paper, our motivation is to use mathematical and simulation approaches to study the throughput performance of the network coding nodes when the link-level SW-ARQ data transmission scheme is employed. As noted previously in Section I, our analytical approaches as well as the derived expressions may be suitable for the network coding nodes employing possibly different coding strategies. Although our study in this paper was inspired by [11] and [12], however, to the best of the authors' knowledge, the work has not been published in the open literature.

Furthermore, we note that our 2ISO model represents an abstraction of the network coding nodes in a variety of applications, such as the network coding nodes employed in distributed antenna systems [18]. It can be seen that our MISO model and the Fork-Join networks [19], [20] share some similarities if we view each of the incoming links of the coding node in our model as a "server" of the Fork-Join networks. However, the Fork-Join network is a one-hop network model consisting of several parallel queues. By contrast, our MISO network considered is a two-hop network, where the first hop consists of several parallel queues as in the Fork-Join network, while the second hop is a first-input-first-service (FIFS) queue with finite buffer. Let us now turn to consider the throughput analysis of the 2ISO network coding node.

\section{Throughrut of Two-InPut-Single-OutPut Network CODING NODE}

\section{A. System Models}

The system considered in this contribution is shown in Fig. 1, which is constituted by three types of nodes, namely source nodes $X_{1}$ and $X_{2}$, network coding node $B$, as well as sink node $C$. Packets to be transmitted are generated at the source nodes $X_{1}, X_{2}$. Node $B$ is a 2ISO node employing packet-level network coding, while the sink node $C$ receives packets from node $B$. As shown in Fig. 1 , link $l_{0}$ connects nodes $B$ and $C$, while links $l_{1}$ and $l_{2}$ connect the source nodes $X_{1}$ and $X_{2}$ with node $B$. We assume that packets are transmitted based on the SW-ARQ strategy by the links $l_{0}, l_{1}$, and $l_{2}$. 
At node $B$ of Fig. $1, b_{0}$ denotes the buffer that stores the outstanding packet being transmitted by $l_{0}$. By contrast, $b_{1}$ and $b_{2}$ are the buffers that store the packets having been successfully received from links $l_{1}$ and $l_{2}$, respectively. In Fig. 1 , the network coding operation is represented by $\oplus$. Note that, although the notation $\oplus$ is used, it does not necessary mean the "XOR" operation. In fact, our study in this contribution is independent of the specific network coding employed and is suitable for any types of network coding operations, provided that the following assumptions adopted in this paper are met.

- The system is operated in synchronous manner.

- The sources $X_{1}$ and $X_{2}$ always have packets to send. The $n$th packets generated by $X_{1}$ and $X_{2}$ are denoted by $x_{1}(n)$ and $x_{2}(n)$, respectively.

- Each of the links $l_{0}, l_{1}$, and $l_{2}$ is divided into two channels: the forward channel and the feedback channel. The forward channel is assumed to be a binary symmetric channel. The probability that a detectable packet error occurs is denoted as $p_{0}, p_{1}$, or $p_{2}$ for the $\operatorname{link} l_{0}, l_{1}$, or $l_{2}$. We assume that the undetectable packet errors can be neglected, which is usually reasonable since, for most error-control codes adopted in practical communication systems, the probability of undetectable errors is very small, in comparison to the probability of detectable errors. Furthermore, we assume that the feedback channel is perfect without yielding transmission errors.

- Let $T$ denote the round-trip time (RTT), which is the time duration between when a node sends a packet and when it receives a confirmation signal. We assume that half of an RTT, i.e., $T / 2$, is required for transmitting a packet from one node to another by the corresponding forward channel. Similarly, half of an RTT is required for sending a confirmation signal from one node to another by the corresponding feedback channel.

- The duration of packets is assumed much shorter than $T$ of the RTT and can be ignored. Furthermore, the processing time of a packet is assumed to be absorbed in the RTT.

- Buffer $b_{0}, b_{1}$, or $b_{2}$ can store only one packet. When both $x_{1}(n)$ and $x_{2}(n)$ are ready and stored in $b_{1}$ and $b_{2}$, once $b_{0}$ is empty, $x_{1}(n)$ and $x_{2}(n)$ are encoded to form $x_{0}(n)$, which is immediately stored into $b_{0}$. At the same time, the buffers $b_{1}$ and $b_{2}$ are released for receiving the following packets.

Based on the above assumptions, the operations at nodes $X_{1}$, $X_{2}, B$, and $C$ at time $t=m T, m=0,1, \ldots$, or $t=(m-$ $(1 / 2)) T, m=1,2, \ldots$, can be described as follows.

1) First, at $t=0$, the source nodes $X_{1}$ and $X_{2}$ transmit $x_{1}(0)$ and $x_{2}(0)$ through respective links $l_{1}$ and $l_{2}$ to node $B$. Correspondingly, the outstanding packet is set to $x_{1}(0)$ for source node $X_{1}$ and $x_{2}(0)$ for source node $X_{2}$.

2) Assume that a packet $x_{1}(n)$ (or $\left.x_{2}(n)\right)$ is transmitted by node $X_{1}\left(\right.$ or $\left.X_{2}\right)$ to node $B$ at time $t=(m-1) T$, $m=1,2, \ldots$. This packet arrives at node $B$ through the forward channel of link $l_{1}\left(\right.$ or $\left.l_{2}\right)$ at time $t=(m-(1 / 2)) T$ after half of an RTT. Upon receiving this packet, node $B$ checks whether the packet $x_{1}(n)$ (or $x_{2}(n)$ ) is corrupted during the transmission and whether the buffer $b_{1}$ (or $b_{2}$ ) is available to store. If the received packet is corrupted or the buffer $b_{1}$ (or $b_{2}$ ) is still occupied by the last packet $x_{1}(n-1)$ (or $x_{2}(n-1)$ ), a NACK is sent back through the feedback channel of link $l_{1}$ (or $l_{2}$ ) to $X_{1}$ (or $X_{2}$ ). Otherwise, node $B$ sends an ACK to node $X_{1}$ (or $X_{2}$ ) and simultaneously stores $x_{1}(n)$ (or $x_{2}(n)$ ) into buffer $b_{1}$ (or $b_{2}$ ).

In the context of the sink node $C$, if there is a packet $x_{0}(n)$ transmitted by node $B$ at $t=(m-1) T$, this packet is received by node $C$ from the forward channel of link $l_{0}$ at time $t=(m-(1 / 2)) T$. In this case, the outstanding packet of node $B$ is set to $x_{0}(n)$, which is stored temporarily in buffer $b_{0}$. Upon receiving this packet, node $C$ checks whether the packet is corrupted during the transmission. If the packet is corrupted, a NACK is fed back to node $B$. By contrast, if the packet is assumed correct, then $x_{0}(n)$ is accepted by node $C$, and node $C$ sends an ACK to node $B$ using the feedback channel of link $l_{0}$.

3) At $t=m T$, source node $X_{1}$ (or $X_{2}$ ) and coding node $B$ check the feedback channels to see whether the last transmitted packets are successfully conveyed. Specifically, for the source node $X_{1}$ (or $X_{2}$ ), if an ACK is received from the feedback channel of link $l_{1}$ (or $l_{2}$ ) while the outstanding packet is $x_{1}(n)$ (or $x_{2}(n)$ ), then source node $X_{1}$ (or $X_{2}$ ) transmits the next packet $x_{1}(n+1)\left(\right.$ or $\left.x_{2}(n+1)\right)$. Simultaneously, the outstanding packet for $X_{1}$ (or $\left.X_{2}\right)$ is set to $x_{1}(n+1)$ (or $\left.x_{2}(n+1)\right)$. However, if a NACK is received from the feedback channel of link $l_{1}$ (or $l_{2}$ ) while the outstanding packet for source node $X_{1}$ (or $X_{2}$ ) is $x_{1}(n)$ (or $x_{2}(n)$ ), then the packet $x_{1}(n)$ (or $x_{2}(n)$ ) is retransmitted to node $B$ through the forward channel of link $l_{1}$ (or $l_{2}$ ).

For the coding node $B$, if an ACK is received from the feedback channel of link $l_{0}$ while the outstanding packet stored in $b_{0}$ is $x_{0}(n)$, then node $B$ updates the outgoing packet from $x_{0}(n)$ to $x_{0}(n+1)$ by encoding the contents of $b_{1}$ and $b_{2}$. Simultaneously, $x_{0}(n+1)$ is transmitted from node $B$ to node $C$ through the forward channel of link $l_{0}$. By contrast, if a NACK is received from the feedback channel of link $l_{0}$ while the outstanding packet stored in $b_{0}$ is $x_{0}(n)$, then node $B$ retransmits $x_{0}(n)$ over the forward channel of link $l_{0}$ and retains $x_{0}(n)$ as its outstanding packet.

According to our above discussion, explicitly, the system model of Fig. 1 fits well the coding path in the butterfly network, as shown by the heavily lined path in Fig. 2, associated with the following assumptions.

- The two links from source nodes $X_{1}$ and $X_{2}$ to node $B$ are two links that employ end-to-end SW-ARQ transmission scheme associated with an RTT of $T$.

- Node $C$ has an infinite buffer capacity for storing received packets. In other words, node $C$ rejects a received packet only if the packet is detected in error.

As we know from [1] and [3], in the butterfly network of Fig. 2, the data $\left\{x_{1}(n)\right\}$ and $\left\{x_{2}(n)\right\}$ generated at the source nodes $X_{1}$ and $X_{2}$, respectively, are required to be sent to both the sink nodes $S_{1}$ and $S_{2}$. Specifically, when we consider the sink node $S_{1}$, the data $\left\{x_{1}(n)\right\}$ generated at the source node $X_{1}$ can be directly sent to this sink node without the constraint from the network coding node $B$. Hence, the corresponding 


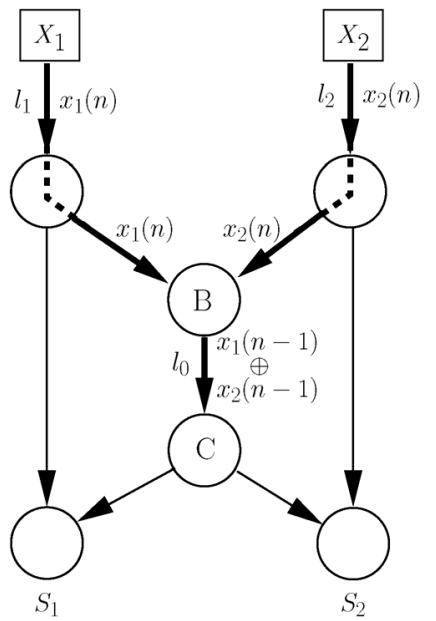

Fig. 2. Schema of butterfly network. It can be seen that the two-input-oneoutput coding node model shown in Fig. 1 forms the coding path of the butterfly network.

throughput may not be affected by the throughput of the coding node $B$. However, as seen in Fig. 2 , the data $\left\{x_{2}(n)\right\}$ sent from the source node $X_{2}$ to the sink node $S_{1}$ goes through the network coding node $B$, and therefore, the resultant throughput is limited by the network coding node. Furthermore, if we assume that all links in the network have a similar reliability, then we have reason to assume that the throughput of the direct link from $X_{1}$ to $S_{1}$ is higher than that of the coding path containing node $B$. This is because successful delivery of a packet by the coding node $B$ depends on that it correctly receives two packets from both the source nodes $X_{1}$ and $X_{2}$. Consequently, if transmitting data from $X_{1}$ and $X_{2}$ to $S_{1}$ is the ultimate objective, the overall throughput at node $S_{1}$ is the sum of the direct link's throughput and the coding path's throughput. However, if $S_{1}$ is also an intermediate node and if its following operations depend on both $\left\{x_{1}(n)\right\}$ and $\left\{x_{2}(n)\right\}$, then the achievable throughput at node $S_{1}$ is limited by the coding path's throughput. Additionally, from Fig. 2, we can easily know that analyzing the throughput of the coding path is much more challenging than analyzing the throughput of the direct links, which is a classic problem of throughput analysis. Therefore, it is highly important for us to study separately the throughput of network coding node.

\section{B. State Machine Modeling of Operations at Network Coding Node}

In this section, we first show that a finite state machine can be employed to represent the network coding system shown in Fig. 1. Then, we illustrate that this finite state machine is a stationary Markov process. Therefore, the steady-state throughput of the system is analyzed, where the steady-state throughput is defined as the rate of the packets received by the node $C$ from the coding node $B$.

Let us first analyze the throughput of node $B$. Let $Q=\{0,1\}$ be a set containing the two states of $b_{i}, i=0,1,2$, where state " 1 " corresponds to the state that $b_{i}$ is available to store a new packet, while state " 0 " indicates that $b_{i}$ is occupied. Then, the state of node $B$ at every $t=m T$ is determined by the
TABLE I

List of STATES OF THE Coding Node $B$

\begin{tabular}{|c|c||c|c|}
\hline State & $S(m)$ & State & $S(m)$ \\
\hline$S_{0}$ & $(0,0,0)$ & $S_{4}$ & $(1,0,0)$ \\
$S_{1}$ & $(0,0,1)$ & $S_{5}$ & $(1,0,1)$ \\
$S_{2}$ & $(0,1,0)$ & $S_{6}$ & $(1,1,0)$ \\
$S_{3}$ & $(0,1,1)$ & $S_{7}$ & $(1,1,1)$ \\
\hline
\end{tabular}

triple $S(m)=\left[q_{2}(m), q_{1}(m), q_{0}(m)\right]$, where $q_{i}(m) \in Q$ for $i=0,1,2$. Let $S_{0}, S_{1}, \ldots, S_{7}$ denote the states of node $B$ corresponding to all the possible combinations of the triple $S(m)=$ $\left[q_{2}(m), q_{1}(m), q_{0}(m)\right]$, which are listed in Table I.

As an example, Fig. 3 demonstrates the state transition of node $B$ with the time, where the number in every box represents the generation number of the corresponding packet, i.e., the number indicating which batch the packet comes from. In more detail, Fig. 3 can be explained as follows.

1) Let us assume that at $t=0$, the state of node $B$ is $S(0)=$ $S_{7}$. At $t=0$, source nodes $X_{1}$ and $X_{2}$ send packets $x_{1}(1)$ and $x_{2}(1)$ to node $B$.

2) At $t=T / 2, x_{1}(1)$ and $x_{2}(1)$ are received by node $B$ without any errors and, hence, $x_{1}(1)$ and $x_{2}(1)$ are stored in $b_{1}$ and $b_{2}$, and node $B$ sends correspondingly the ACKs to nodes $X_{1}$ and $X_{2}$. In this case, the state of node $B$ at $t=T / 2$ changes to $S(1 / 2)=S_{1}$.

3) At $t=T$, node $B$ encodes $x_{1}(1)$ and $x_{2}(1)$ into $x_{0}(1)$, which is stored into $b_{0}$ and is also sent to node $C$. Correspondingly, the state of node $B$ is set to $S(1)=S_{6}$. At $t=T$, source nodes $X_{1}$ and $X_{2}$ receive the ACKs for $x_{1}(1)$ and $x_{2}(1)$. Hence, they send $x_{1}(2)$ and $x_{2}(2)$ to node $B$ at $t=T$.

4) As seen in Fig. 3 , at $t=1.5 T, x_{2}(2)$ is received by node $B$ without error, but $x_{1}(2)$ is received in error. Hence, node $B$ sends node $X_{2}$ an ACK for $x_{2}(2)$, but sends node $X_{1}$ a NACK for requesting a retransmission of $x_{1}(2)$. Correspondingly, the state of node $B$ becomes $S(1.5)=S_{2}$. Furthermore, it can be implied from Fig. 3 that transmission errors are detected in $x_{0}(1)$ by node $C$. Thus, node $C$ sends back node $B$ a NACK to request retransmission of $x_{0}(1)$ at $t=1.5 T$.

5) At $t=2 T$, node $B$ receives a NACK for $x_{0}(1)$ from node $C$ and retransmits $x_{0}(1)$ over the forward channel of $l_{0}$. At the same time, node $X_{2}$ receives an ACK for $x_{2}(2)$, while node $X_{1}$ receives a NACK for $x_{1}(2)$ from node $B$. Hence, node $X_{2}$ transmits a new packet $x_{2}(3)$ over the forward channel of link $l_{2}$, and node $X_{1}$ retransmits $x_{1}(2)$ over the forward channel of link $l_{1}$. At $t=2 T$, the state of node $B$ remains unchanged and is $S(2)=S_{2}$.

6) At $t=2.5 T, x_{2}(3)$ arrives at node $B$ without error, but finds $b_{2}$ is occupied. Thus, node $B$ sends a NACK along the feedback channel of link $l_{2}$. At the same time, $x_{1}(2)$ is received by node $B$ without error. Therefore, node $B$ sends an ACK to node $X_{1}$ and stores $x_{1}(2)$ into $b_{1}$, making the state of $b_{1}$ change from 1 to 0 . Furthermore, at $t=2.5 T$, $x_{0}(2)$ arrives at node $C$ without error. Therefore, node $C$ sends an ACK to node $B$ using the feedback channel of $l_{0}$. From the above analysis, it can be seen that the state of node $B$ at $t=2.5 T$ is $S(2.5)=S_{0}$. 


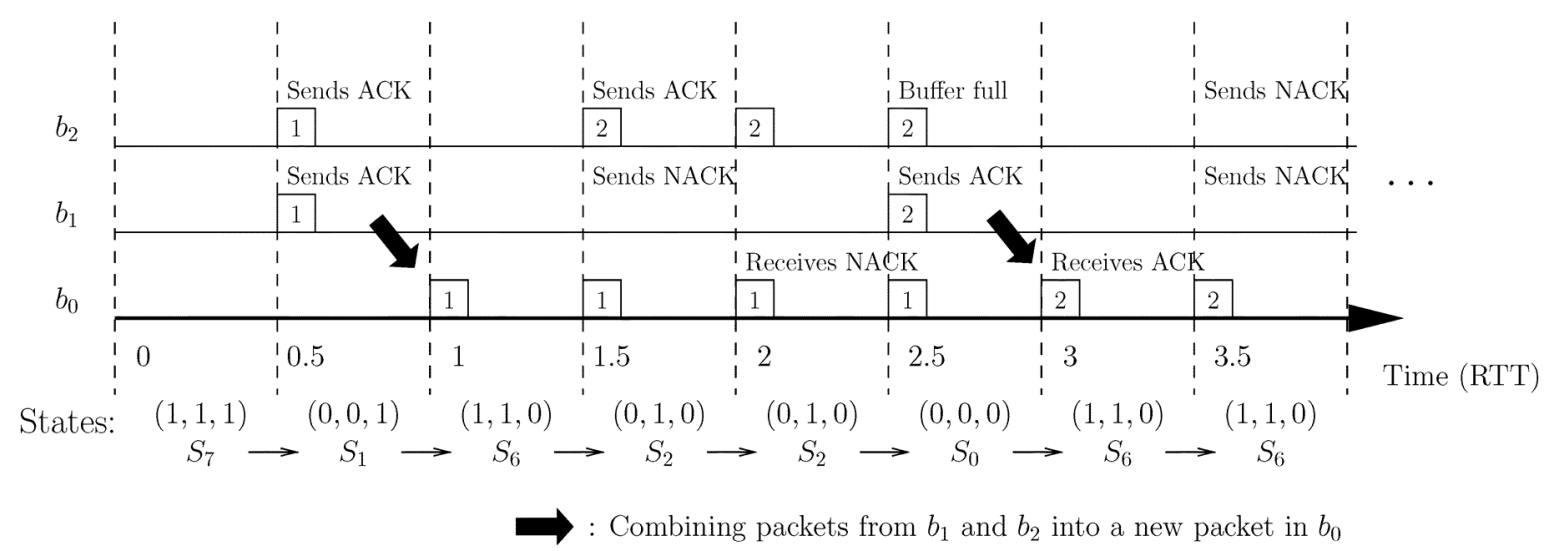

Fig. 3. Illustration of state transition of node $B$.

7) At $t=3 T$, node $X_{2}$ receives a NACK for $x_{2}(3)$, and node $X_{1}$ receives a ACK for $x_{1}(2)$, hence node $X_{2}$ retransmits $x_{2}(3)$, and node $X_{1}$ transmits a new packet $x_{1}(3)$ over the forward channels of links $l_{2}$ and $l_{1}$, respectively. At $t=3 T$, node $B$ receives an ACK for $x_{0}(1)$ from the feedback channel of link $l_{0}$. Hence, node $B$ encodes $x_{2}(2)$ and $x_{1}(2)$ into $x_{0}(2)$ and then stores it into $b_{0}$ as well as sends it to node $C$. Based on the above analysis, the state of node $B$ at $t=3 T$ is $S(3)=S_{6}$.

8) As shown in Fig. 3 , at $t=3.5 T$, both $x_{2}(3)$ and $x_{1}(3)$ are detected by node $B$ with transmission errors. Hence, both packets are rejected by node $B$, and the states of $b_{2}$ and $b_{1}$ do not change. Since $b_{0}$ is still occupied by $x_{0}(2)$ at $t=$ $3.5 T$, the state of node $B$ at $t=3 T$ is still $S(3.5)=S_{6}$.

The state of node $B$ at other times can be analyzed in the same way as discussed above. Explicitly, the network coding operations at node $B$ can be described by a state machine with eight states. Let us analyze the steady-state throughput of the coding node $B$. Note that, in this paper, the normalized throughput is considered. Given that $N(m)$ packets are successfully conveyed from node $B$ to node $C$ during $t=0$ and $t=m T$, the normalized throughput is defined as

$$
R(m)=\frac{N(m)}{m}, \quad m=1,2, \ldots
$$

When $m \rightarrow \infty, R(m)$ converges to the normalized steadystate throughput expressed by $R$. Furthermore, it can be readily shown that the steady-state throughput $R$ is equal to the probability that coded packets are formed and forwarded to $b_{0}$ by node $B$.

\section{Throughput Analysis}

From the analysis in Section III-B, we can know that the throughput analysis of node $B$ can be carried out by considering only the integer values of $m$. This is because, as seen in Fig. 3 and the corresponding description, a packet can possibly be successfully delivered at $t=m T$ only when $m$ is an integer. Furthermore, as demonstrated in Section III-B, when $m$ is an integer, the state of node $B$ will never enter state $S_{1}$, which can be explained in detail as follows. Let us assume that the state of node $B$ at time $t=m T$ is $S(m)=S_{1}$. Then, according to the operation procedure described in Section III-A, we must have $q_{1}(m-1 / 2)=0$ and $q_{2}(m-1 / 2)=0$. However, $q_{0}(m-1 / 2)$ only takes value " 0 " or " 1 ," which results in the following cases.

1) $q_{0}(m-(1 / 2))=1$ : Since $q_{0}(m-1 / 2)=1$, then at $t=m T$, packets in $b_{1}$ and $b_{2}$ should be encoded into a new packet and stored into $b_{0}$, making $q_{0}(m)=0$. Simultaneously, both the buffers of $b_{1}$ and $b_{2}$ are released for receiving the following packets, yielding $q_{1}(m)=$ $1, q_{2}(m)=1$. Hence, the state of node $B$ at $t=m T$ should be $S(m)=S_{6}$, which contradicts the assumption of $S(m)=S_{1}$.

2) $q_{0}(m-(1 / 2))=0$, and node $B$ receives an ACK at $t=m T$ : In this case, buffer $b_{0}$ is released at $t=m T$. The packets in $b_{1}$ and $b_{2}$ are encoded to form a new packet and stored into $b_{0}$, making $q_{0}(m)=0$. Since both $b_{1}$ and $b_{2}$ are available for receiving the following packets, we have $q_{1}(m)=1, q_{2}(m)=1$. Hence, the state of node $B$ at $t=m T$ is $S(m)=S_{6}$ instead of $S(m)=S_{1}$ as assumed.

3) $q_{0}(m-(1 / 2))=0$, and node $B$ receives a NACK at $t=m T$ : In this case, node $B$ needs to retransmit the packet stored in $b_{0}$ and $q_{0}(m)=0$. Furthermore, $q_{1}(m)=$ $0, q_{2}(m)=0$, since both $b_{1}$ and $b_{2}$ cannot be released at $t=m T$. Hence, the state of node $B$ in this case is $S(m)=S_{0}$, which again contradicts the assumption of $S(m)=S_{1}$.

From the above analysis, it can be implied that, when $q_{1}(m-$ $1 / 2)=0$ and $q_{2}(m-1 / 2)=0, q_{0}(m)=0$ for any cases, implying that $b_{0}$ is always occupied at $t=m T$. Therefore, in our analysis below, we assume that $m$ is an integer and do not consider the state $S_{1}$.

Let $P_{i}(m)$ denote the probability that the state of node $B$ is $S_{i}$ at time $t=m T$. Let $\boldsymbol{p}(m)=\left[P_{0}(m), P_{2}(m), \cdots, P_{7}(m)\right]^{T}$, which does not contain $P_{1}(m)$ since $P_{1}(m)$ is always zero. Explicitly, we have $\sum_{i=0.2}^{7} P_{i}(m)=1$. Let us assume that the system starts with an idle state $S(0)=S_{7}$. Then, we have

$$
\boldsymbol{p}(0)=[0,0,0,0,0,0,1]^{T} .
$$

Let $P_{i, j}(m)=P\left[S(m+1)=S_{j} \mid S(m)=S_{i}\right], i, j=$ $0,2, \cdots, 7$, denote the transition probability from state $S_{i}$ at $t=m T$ to state $S_{j}$ at $t=(m+1) T . P_{i, j}(m)$ can be derived based on the buffer states in $S_{i}$ and $S_{j}$ as well as the probabilities that links $l_{0}, l_{1}$, and $l_{2}$ successfully deliver their packets. 
Specifically, let $D_{i}, i=0,1,2$, denote the event that $\operatorname{link} l_{i}$ successfully delivers a packet and $P\left[D_{i}\right]=\bar{p}_{i}$, while $\bar{D}_{i}$ denote the event that $l_{i}$ fails to deliver a packet on link $l_{i}$ and $P\left[\bar{D}_{i}\right]=p_{i}$. Explicitly, $p_{i}=1-\bar{p}_{i}$. Then, it can be shown that we have

$$
\begin{aligned}
P_{0,0}(m)= & P\left[S(m+1)=S_{0} \mid S(m)=S_{0}\right] \\
= & P\left[\bar{D}_{0}\right]=p_{0} \\
P_{0,2}(m)= & P\left[S(m+1)=S_{2} \mid S(m)=S_{0}\right] \\
= & 0 \text { (illegitimate transition) } \\
& \vdots \\
P_{4,5}(m)= & P\left[S(m+1)=S_{5} \mid S(m)=S_{4}\right] \\
= & P\left[D_{0}\right]\left(P\left[D_{1}\right]+P\left[\bar{D}_{1}\right]\right) P\left[\bar{D}_{2}\right]=\bar{p}_{0} p_{2} \\
& \vdots \\
P_{6,6}(m)= & P\left[S(m+1)=S_{6} \mid S(m)=S_{6}\right] \\
= & P\left[D_{0}\right] P\left[D_{1}\right] P\left[D_{2}\right]+P\left[\bar{D}_{0}\right] P\left[\bar{D}_{1}\right] P\left[\bar{D}_{2}\right] \\
= & \bar{p}_{0} \bar{p}_{1} \bar{p}_{2}+p_{0} p_{1} p_{2} \\
& \vdots \\
P_{7,7}(m)= & P\left[S(m+1)=S_{7} \mid S(m)=S_{7}\right] \\
= & P\left[\bar{D}_{1}\right] P\left[\bar{D}_{2}\right]=p_{1} p_{2} .
\end{aligned}
$$

As an example, we explain how $P_{4,5}(m)$ is obtained. Note that since $i, j \neq 1, P_{4,5}(m)$ is the $(4,5)$ th element of $\mathbf{P}$ seen in (4) at the bottom of the page. For $P_{4,5}(m)$, we have $S(m)=[100]$ and $S(m+1)=[101]$, which means that a packet in $b_{0}$ is successfully delivered to node $C$, yielding a probability $P\left[D_{0}\right]=\bar{p}_{0}$, while the link $l_{2}$ fails to deliver $b_{2}$ a packet, corresponding to a probability $P\left[\bar{D}_{2}\right]=p_{2}$. Furthermore, since there is a packet occupying $b_{1}$, it cannot accept a new packet no matter whether this packet is correct or not. Hence, this transition probability is $P\left[D_{1}\right]+P\left[\bar{D}_{1}\right]=1$. Consequently, we have $P_{4.5}(m)=$ $P\left[D_{0}\right]\left(P\left[D_{1}\right]+P\left[\bar{D}_{1}\right]\right) P\left[\bar{D}_{2}\right]=\bar{p}_{0} p_{2}$. From the above equations in (3), we can see that $\left\{P_{i, j}(m)\right\}$ 's are independent of $m$, implying that $\left\{P_{i, j}(m)\right\}$ are time-invariant. Therefore, in our forthcoming discourse, the index $m$ associated with the transition probabilities is dropped for simplicity. Finally, with the aid of (3), it can be shown that the transition matrix $\mathbf{P}$ can be expressed as (4).

Using the law of total probability [24], the probability $P_{j}(m+1)$ can be expressed as

$$
P_{j}(m+1)=\sum_{i=0,2}^{7} P_{i, j} P_{i}(m), \quad j=0,2, \ldots, 7
$$

which, when considering all the seven states, can be expressed in vector form as

$$
\boldsymbol{p}(m+1)=\boldsymbol{P}^{T} \boldsymbol{p}(m) .
$$

Equation (6) represents a recursive equation. Hence, it can be readily shown that $\boldsymbol{p}(m)$ can be expressed as

$$
\boldsymbol{p}(m)=\left(\boldsymbol{P}^{T}\right)^{m} \boldsymbol{p}(0), \quad m=0,1, \ldots
$$

As shown in (4), the sum of each row of $\mathbf{P}$ is equal to one. Hence, $\mathbf{P}^{T}$ is a left stochastic matrix [24], whose limit of $\lim _{m \rightarrow \infty}\left(\mathbf{P}^{T}\right)^{m}$ exists, according to the Perron-Frobenius theorem [25], [24]. Therefore, when $m \rightarrow \infty$, the Markov process becomes stationary [26] and yields

$$
\boldsymbol{p}(m+1)=\boldsymbol{p}(m) .
$$

Let $\boldsymbol{\pi}=\left[\pi_{0}, \pi_{2}, \cdots, \pi_{7}\right]^{T}=\lim _{m \rightarrow \infty} \boldsymbol{p}(m)$. Then, the steadystate probabilities in $\pi$ can be obtained by solving the equation

$$
\boldsymbol{\pi}=\boldsymbol{P}^{T} \boldsymbol{\pi}
$$

associated with the constraint $\sum_{i=0,2}^{7} \pi_{i}=1$. Equation (9) shows that $\boldsymbol{\pi}$ is the right eigenvector of matrix $\boldsymbol{P}^{T}$ associated with an eigenvalue one. Therefore, $\boldsymbol{\pi}$ can be evaluated with the aid of the methods for solving the eigenvector problem [24], [25].

Finally, when reaching the steady-state, the throughput of the 2ISO network coding node of Fig. 1 or of the 2ISO network coding branch of Fig. 2 is the rate that the encoded packets are successfully conveyed from node $B$ to node $C$. This rate is also equal to the rate that packets in $b_{1}$ and $b_{2}$ are encoded and forwarded to $b_{0}$. When this rate is normalized by $T$ of the RTT, the throughput is then equal to the probability that the packets are forwarded from nodes $b_{1}$ and $b_{2}$ to $b_{0}$.

From the operation principles described in Section III-A, we can know that the state $S_{i}, i \neq 6$, say, at $t=m T$ always changes to $S_{6}$ at $t=(m+1) T$, when the packets in $b_{1}$ and $b_{2}$ are encoded and forwarded to $b_{0}$ at $t=(m+1) T$. Otherwise, the state at $t=(m+1) T$ will never be $S_{6}$. By contrast, when $S(m)=S_{6}$, then $S(m+1)=S_{6}$ either if all the packets transmitted at $t=m T$ are correctly conveyed - which results in that the two packets received by node $B$ are encoded and forwarded to $b_{0}$ at $t=(m+1) T$, and this probability is $\bar{p}_{0} \bar{p}_{1} \bar{p}_{2}$ - or if all the packets transmitted at $t=m T$ are detected in error, which makes node $B$ retains its previous state $S_{6}$. However, only the former event makes contribution to the throughput. Therefore,

$$
\boldsymbol{P}=\left[\begin{array}{ccccccc}
p_{0} & 0 & 0 & 0 & 0 & \bar{p}_{0} & 0 \\
p_{0} \bar{p}_{1} & p_{0} p_{1} & \bar{p}_{0} p_{1} & 0 & 0 & \bar{p}_{0} \bar{p}_{1} & 0 \\
0 & 0 & p_{1} & 0 & 0 & \bar{p}_{1} & 0 \\
p_{0} \bar{p}_{2} & 0 & 0 & p_{0} p_{2} & \bar{p}_{0} p_{2} & \bar{p}_{0} \bar{p}_{2} & 0 \\
0 & 0 & 0 & 0 & p_{2} & \bar{p}_{2} & 0 \\
p_{0} \bar{p}_{1} \bar{p}_{2} & p_{0} p_{1} \bar{p}_{2} & \bar{p}_{0} p_{1} \bar{p}_{2} & p_{0} \bar{p}_{1} p_{2} & \bar{p}_{0} \bar{p}_{1} p_{2} & \bar{p}_{0} \bar{p}_{1} \bar{p}_{2}+p_{0} p_{1} p_{2} & \bar{p}_{0} p_{1} p_{2} \\
0 & 0 & p_{1} \bar{p}_{2} & 0 & \bar{p}_{1} p_{2} & \bar{p}_{1} \bar{p}_{2} & p_{1} p_{2}
\end{array}\right]
$$


when considering all the above events, the throughput of the 2ISO network coding node normalized by $T$ of the RTT can be expressed as

$$
\begin{aligned}
R= & P_{0}(\infty) P_{0,6}+P_{2}(\infty) P_{2,6}+P_{3}(\infty) P_{3,6}+P_{4}(\infty) P_{4,6} \\
& +P_{5}(\infty) P_{5,6}+P_{6}(\infty) \bar{p}_{0} \bar{p}_{1} \bar{p}_{2}+P_{7}(\infty) P_{7,6} \\
= & \pi_{0} P_{0,6}+\pi_{2} P_{2,6}+\pi_{3} P_{3,6}+\pi_{4} P_{4,6}+\pi_{5} P_{5,6} \\
& +\pi_{6} \bar{p}_{0} \bar{p}_{1} \bar{p}_{2}+\pi_{7} P_{7,6}
\end{aligned}
$$

where $\pi_{i} P_{i, 6}, i \neq 6$, is the probability of the event that the current state is $S_{i}$, which transits to $S_{6}$ at the next time.

From the discussion and analysis in this section, it can be implied that our analytical approaches and the derived expressions may be applied for the network coding nodes with different network coding schemes, provided that, in these network coding schemes, a network coding node requires one packet from each of the incoming links before encoding a new outgoing packet. Note furthermore that our approaches and derived expressions are also suitable for the node employing random network coding. This is because, in our model, every buffer associated with the network coding node is assumed to store at most one packet. In this case, when operated under the SW-ARQ, the network coding always deals with the packets of the same generation of all the source nodes, regardless of the random network coding scheme. Let us now consider the case of network coding nodes having multiple incoming channels.

\section{Throughput of Multiple-InPut-Single-Output NeTWORK CODING NODE}

In this section, we extend our study in Section III to the MISO network coding nodes, as shown, for example, in the diagram of Fig. 4. As with Fig. 1 for the 2ISO system, the MISO network coding node of Fig. 4 is also constituted by three types of nodes, namely, source nodes $X_{1}, X_{2}, \ldots, X_{H}$, network coding node $B$, and sink node $C$. As shown in Fig. 4, the source nodes $X_{1}, X_{2}, \ldots, X_{H}$ are connected with the coding node $B$ through the $H$ links, $l_{1}, l_{2}, \ldots, l_{H}$. The sink node $C$ is connected with the coding node $B$ via one link $l_{0}$. We assume that each link in Fig. 5 consists of a forward channel and a feedback channel. Furthermore, in Fig. $4, b_{0}$ and $b_{1}, b_{2}, \ldots, b_{H}$ are the buffers for storing the outstanding packet transmitted on link $l_{0}$ and the packets received from links $l_{1}, l_{2}, \ldots, l_{H}$, respectively. It is assumed that each buffer can store at most one packet.

As for the 2ISO network coding node $B$ in Fig. 4, the operations of the MISO network coding node $B$ in Fig. 4 can also be modeled by a Markov chain with $\left(2^{H+1}-1\right)$ states and a corresponding transition matrix $\boldsymbol{P}$. The throughput of the MISO network coding node can also be analyzed in the same way as that for the 2ISO network coding node, once the state transition matrix $\boldsymbol{P}$ is available. Hence, in order to analyze the throughput of the MISO network coding node, it is essential to determine first the state transition matrix $\boldsymbol{P}$. In this paper, an algorithm is proposed for generating the state transition matrix $\boldsymbol{P}$, which is described in detail as follows.

Let $S(m)=\left[q_{H}(m), q_{H-1}(m), \cdots, q_{0}(m)\right]$ denote the state at time $t=m T$, where $q_{h}(m)=1$ or 0 , and $h=0,1, \ldots, H$, means that $b_{h}$ is empty $\left(q_{h}(m)=1\right)$ or occupied $\left(q_{h}(m)=0\right)$

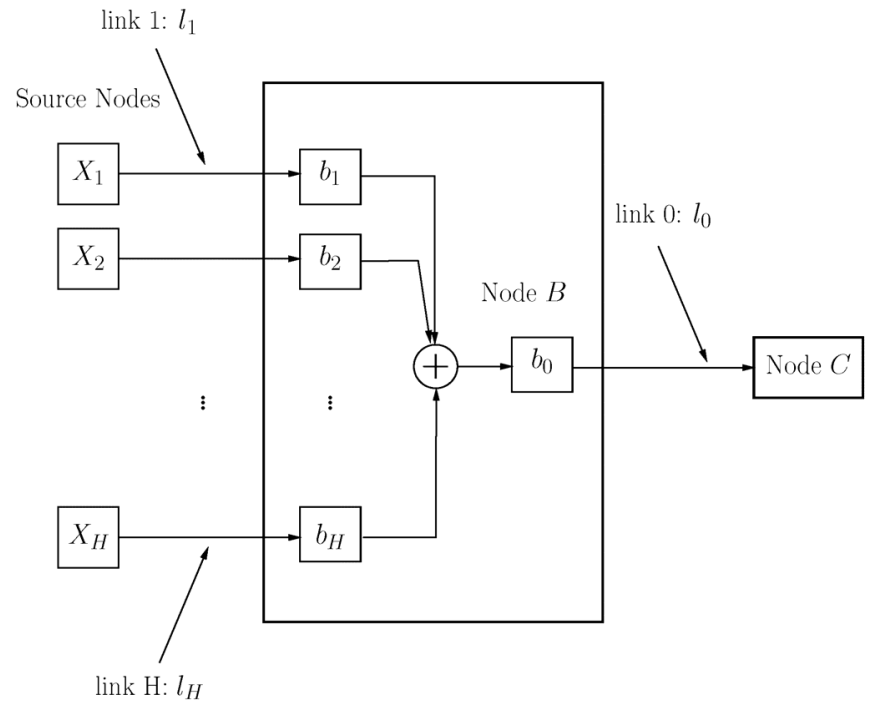

Fig. 4. Network coding node $B$ with $H$ incoming links $l_{1}, l_{2}, \ldots, l_{H}$ and one outgoing link $l_{0}$.

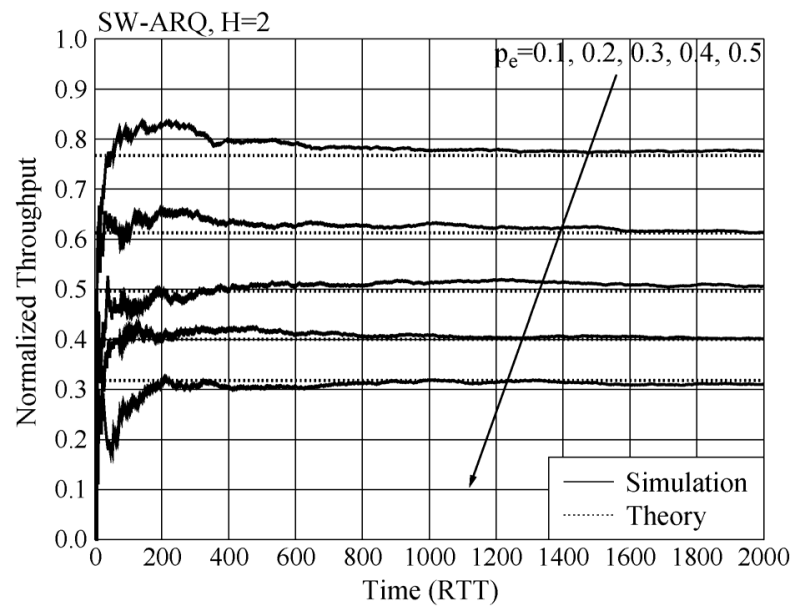

Fig. 5. Steady-state throughput and simulated throughput for the network coding node shown in Fig. 4 with $H=2$. Explicitly, the simulated throughput converges to the theoretical steady-state throughput.

at $t=m T$. It can be shown that, for the MISO network coding node, the state $S(m)=[0,0, \ldots, 0,1]$ does not exist, which explains that the Markov chain has $\left(2^{H+1}-1\right)$ states. Let the $\left(2^{H+1}-1\right)$ possible states of $S(m)$ be expressed as $S_{0}, S_{2}, \ldots, S_{2^{H+1}-1}$, where the subscript $i$ is the integer representation of a corresponding binary representation of $S(m)=\left[q_{H}(m), q_{H-1}(m), \cdots, q_{0}(m)\right]$, such as $S_{0}=[0,0, \ldots, 0], S_{2^{H+1}-1}=[1,1, \ldots, 1]$, etc.

Let the $\left(2^{H+1}-1\right) \times\left(2^{H+1}-1\right)$-dimensional transition matrix be $\boldsymbol{P}=\left[P_{i, j}\right], i, j=0,2, \ldots, 2^{H+1}-1$, where $P_{i, j}=$ $P_{i, j}(m)=P\left[S(m+1)=S_{j} \mid S(m)=S_{i}\right]$ expresses the transition probability from state $S_{i}$ at $t=m T$ to state $S_{j}$ at $t=(m+1)$. Let $S_{\hat{J}}=(1,1, \cdots, 1,0)$, where $\hat{J}=2^{(H+1)}-2$. Then, the calculation of $P_{i, j}$ can be described as follows.

First, $P_{\hat{J}, \hat{J}}$ corresponding to the transition probability from $S(m)=S_{\hat{J}}$ to $S(m+1)=S(m)=S_{\hat{J}}$ is an exceptional case that needs to be considered specifically. The state changes from $S(m)=S_{\hat{J}}$ to $S(m+1)=S(m)=S_{\hat{J}}$, either if all the packets transmitted on the $(H+1)$ links are correctly received, which 
corresponds a probability of $\prod_{k=0}^{H} \bar{p}_{k}$, or if all these packets are failed to deliver, which has a probability of $\prod_{k=0}^{H} p_{k}$. Hence, the transition probability of $P_{\hat{J}, \hat{J}}$ is given by

$$
P_{\hat{J}, \hat{J}}=\prod_{k=0}^{H} p_{k}+\prod_{k=0}^{H} \bar{p}_{k}
$$

Second, the transition probability $P_{i, \hat{J}}, i=0,2, \ldots, \hat{J}-$ $1, \hat{J}+1$, can be expressed in the form of

$$
P_{i, \hat{J}}=\prod_{h=0}^{H} f_{h}, \quad i=0,2, \ldots, \hat{J}-1, \hat{J}+1
$$

where, by definition

$$
\begin{aligned}
& f_{0}= \begin{cases}\bar{p}_{0}, & \text { if } q_{0}(m)=0 \\
1, & \text { if } q_{0}(m)=1\end{cases} \\
& f_{h}=\left\{\begin{array}{ll}
1, & \text { if } q_{h}(m)=0 \\
\bar{p}_{h}, & \text { if } q_{h}(m)=1
\end{array} \quad h \geq 1 .\right.
\end{aligned}
$$

Note that $f_{0}$ may be interpreted as the probability of availability for $b_{0}$ to store the next new packet, and $b_{0}$ is always available $\left(f_{0}=1\right)$ at $t=(m+1) T$ if $q_{0}(m)=1$ and is available with a probability of $f_{0}=\bar{p}_{0}$ at $t=(m+1) T$ if $q_{0}(m)=0$. By contrast, $f_{h}, h \geq 1$, may be interpreted as the probability of availability of a packet at $b_{h}$ that can be encoded and forwarded to $b_{0}$ at $t=(m+1) T$. Explicitly, $f_{h}=1$ if $q_{h}(m)=0$ and $f_{h}=\bar{p}_{h}$ if $q_{h}(m)=1$.

Except for the above cases, all the other entries $\left\{P_{i, j}, j \neq \hat{J}\right\}$ in $\boldsymbol{P}$ correspond to the transitions that do not yield throughput. In these cases, the state of $b_{0}$ is not affected by the states of $b_{1}, b_{2}, \ldots, b_{H}$, implying that there is no correlation between the inputs and output of the network coding node. Furthermore, from the operation of the network coding node as described in the previous sections, the operations of the input links of the network coding node are also independent. Therefore, the probability of a transition of this type can be obtained by the product of the individual state transition probabilities of $b_{0}, b_{1}, \ldots, b_{H}$, due to the fact that the operations associated with their corresponding links are independent. Specifically, the transition probability $P_{i, j}$ with $j \neq \hat{J}$ can be expressed in the form of

$$
\begin{aligned}
P_{i, j}=\prod_{h=0}^{H} f_{h}, \quad i & =0,2, \ldots, \hat{J}+1 ; \\
j & =0,2, \ldots, \hat{J}-1, \hat{J}+1
\end{aligned}
$$

where $f_{0}$ is the corresponding transition probability of $b_{0}$ defined as

$$
f_{0}= \begin{cases}p_{0}, & \text { if } q_{0}(m)=0 \text { and } q_{0}(m+1)=0 \\ \bar{p}_{0}, & \text { if } q_{0}(m)=0 \text { and } q_{0}(m+1)=1 \\ 0, & \text { if } q_{0}(m)=1 \text { and } q_{0}(m+1)=0 \\ 1, & \text { if } q_{0}(m)=1 \text { and } q_{0}(m+1)=1\end{cases}
$$

and, for $h=1,2, \ldots, H$, the corresponding transition probability of $b_{h}$ can be expressed as

$$
f_{h}= \begin{cases}1, & \text { if } q_{h}(m)=0 \text { and } q_{h}(m+1)=0 \\ 0, & \text { if } q_{h}(m)=0 \text { and } q_{h}(m+1)=1 \\ \bar{p}_{h}, & \text { if } q_{h}(m)=1 \text { and } q_{h}(m+1)=0 \\ p_{h}, & \text { if } q_{h}(m)=1 \text { and } q_{h}(m+1)=1\end{cases}
$$

Note that since the state of node $B$ always changes to $S_{\hat{J}}$ after a new packet is formed and forwarded to $b_{0}$, hence the transition from $S_{i}$ to $S_{j}$ with $j \neq \hat{J}$ means no new packet is formed and forwarded to $b_{0}$. When keeping this in mind, it is then not difficult to follow the equations in (15) and (16). For example, when $q_{0}(m)=0$ (occupied) and $q_{0}(m+1)=0$ (occupied), this event occurs only when the packet is not successfully delivered at $t=(m+1) T$. Hence, we have $f_{0}=p_{0}$. As another example, for the case of $h \geq 1$, the event of $q_{h}(m)=1$ (empty) and $q_{h}(m+1)=0$ (occupied) means that $b_{h}$ is filled by a new packet at $t=(m+1) T$. Hence, the probability is $f_{h}=\bar{p}_{h}$.

After the transition probability matrix $\boldsymbol{P}$ is determined, the steady-state probabilities in $\pi$ can be obtained by following the equations from (6)-(9). Finally, the throughput of the MISO network coding node normalized by $T$ of the RTT is the total probability of entering the state $S_{\hat{J}}$ excluding the probability of the erroneous event from the state $S(m)=S_{\hat{J}}$ to the state $S(m+1)=S_{\hat{J}}$. Hence, the normalized throughput can be expressed as

$$
\begin{aligned}
R & =\sum_{i=0,2}^{2^{(H+1)}-1} \pi_{i} P_{i, \hat{J}}-\pi_{\hat{J}} \prod_{h=0}^{H} p_{h} \\
& =\sum_{i=0,2 ; i \neq \hat{J}}^{2^{(H+1)}-1} \pi_{i} P_{i, \hat{J}}+\pi_{\hat{J}} \prod_{h=0}^{H} \bar{p}_{h} .
\end{aligned}
$$

It can be readily shown that, when $H=2$, (17) is reduced to (10), the throughput expression of 2ISO network coding nodes.

Let us demonstrate a range of performance results for characterizing the throughput performance of the network coding node.

\section{Performance Results}

In this section, we illustrate a range of numerical and simulation results in order to characterize the throughput performance of the MISO network coding node and to justify our analytical results obtained in the previous sections. In our simulation and numerical evaluation examples, we assume that the packet error rates of links $l_{0}, l_{1}, \ldots, l_{H}$ are the same and equal to $p_{e}$.

As an example, Table II shows the steady-state probabilities $\boldsymbol{\pi}$ as well as the normalized throughput, which were evaluated using (10), for different packet error rate values. As the results in Table II show, the normalized throughput decreases as the packet error rate of the communication links increases.

Figs. 5-8 depict the normalized throughput versus the time normalized by RTT, when the network coding node employs $H=2,3,4,5$ incoming links, respectively. In these figures, the corresponding steady-state throughput evaluated by (17) are depicted for all the packet error rate values considered.

From the results of Figs. 5-8, we can observe that the simulated throughput starts at $R(0)=0$ since there are no packets 
TABLE II

Steady-State Probabilities $\boldsymbol{\pi}$ and Corresponding Steady-State Throughput Evaluated By (10)

\begin{tabular}{|c|c|c|c|c|c|c|c|c|}
\hline$p_{e}$ & 0.1 & 0.2 & 0.3 & 0.4 & 0.5 & 0.6 & 0.7 & 0.8 \\
\hline$\pi_{0}$ & 0.0705 & 0.1070 & 0.1282 & 0.1418 & 0.1515 & 0.1591 & 0.1656 & 0.1714 \\
$\pi_{2}$ & 0.0070 & 0.0206 & 0.0353 & 0.0489 & 0.0606 & 0.0702 & 0.0778 & 0.0836 \\
$\pi_{3}$ & 0.0705 & 0.1070 & 0.1282 & 0.1418 & 0.1515 & 0.1591 & 0.1656 & 0.1714 \\
$\pi_{4}$ & 0.0070 & 0.0206 & 0.0353 & 0.0489 & 0.0606 & 0.0702 & 0.0778 & 0.0836 \\
$\pi_{5}$ & 0.0705 & 0.1070 & 0.1282 & 0.1418 & 0.1515 & 0.1591 & 0.1656 & 0.1714 \\
$\pi_{6}$ & 0.7676 & 0.6173 & 0.5096 & 0.4279 & 0.3636 & 0.3120 & 0.2699 & 0.2351 \\
$\pi_{7}$ & 0.0070 & 0.0206 & 0.0353 & 0.0489 & 0.0606 & 0.0702 & 0.0778 & 0.0836 \\
\hline$R$ & 0.7669 & 0.6123 & 0.4958 & 0.4005 & 0.3182 & 0.2446 & 0.1773 & 0.1147 \\
\hline
\end{tabular}

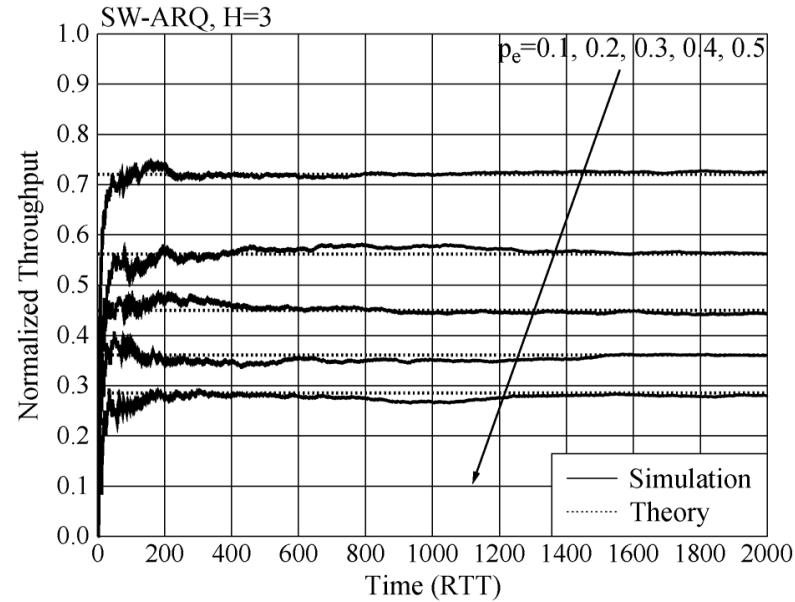

Fig. 6. Steady-state throughput and simulated throughput for the network coding node shown in Fig. 4 with $H=3$. Explicitly, the simulated throughput converges to the theoretical steady-state throughput.

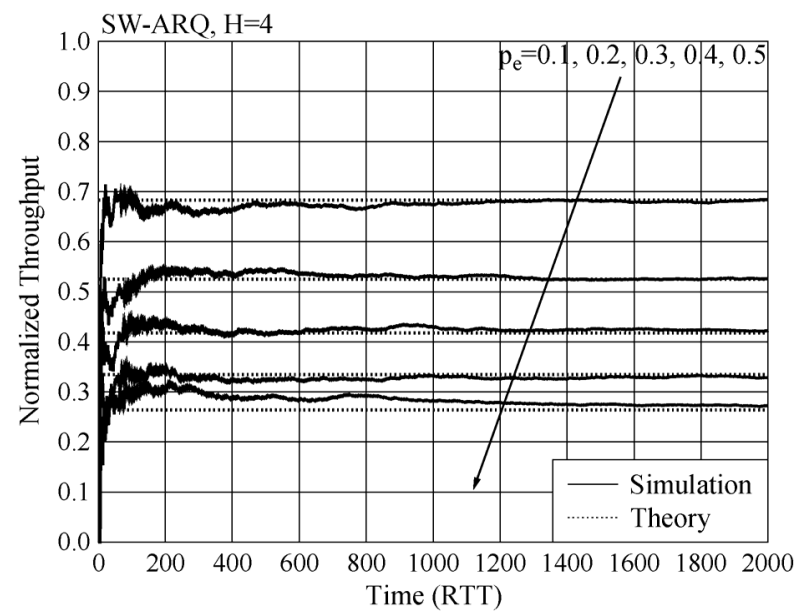

Fig. 7. Steady-state throughput and simulated throughput for the network coding node shown in Fig. 4 with $H=4$. Explicitly, the simulated throughput converges to the theoretical steady-state throughput.

received by node $C$ at $t=0$. Then, the throughput obtained by simulations fluctuates around its corresponding steady-state throughput obtained from evaluation of (17) due to insufficient number of samples. Finally, the throughput obtained by simulations converges to the theoretical steady-state throughput. The results of Figs. 5-8 demonstrate that our analytical results obtained in Sections III and IV are effective for evaluation of the steady-state throughput of MISO network coding nodes.

Finally, in Fig. 9, we compare the normalized steady-state throughput versus packet error rate performance of the network

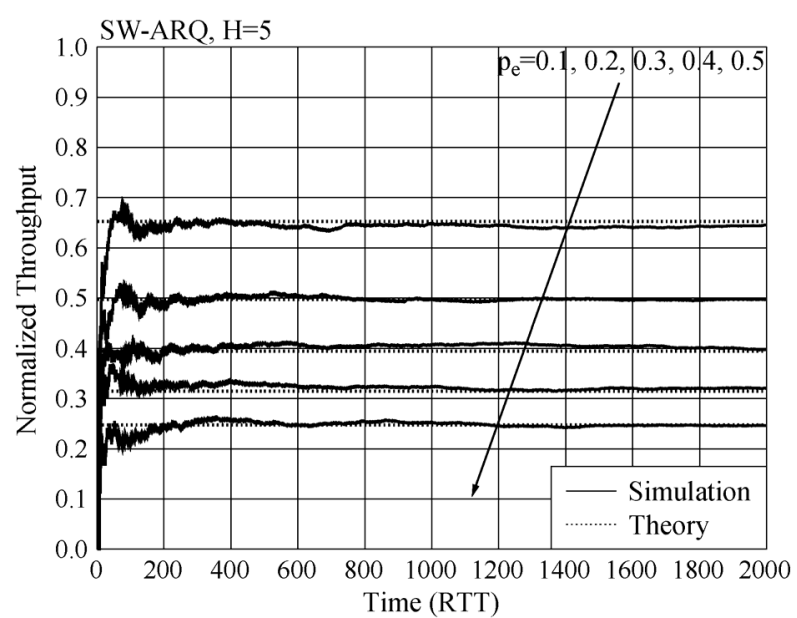

Fig. 8. Steady-state throughput and simulated throughput for the network coding node shown in Fig. 4 with $H=5$. Explicitly, the simulated throughput converges to the theoretical steady-state throughput.

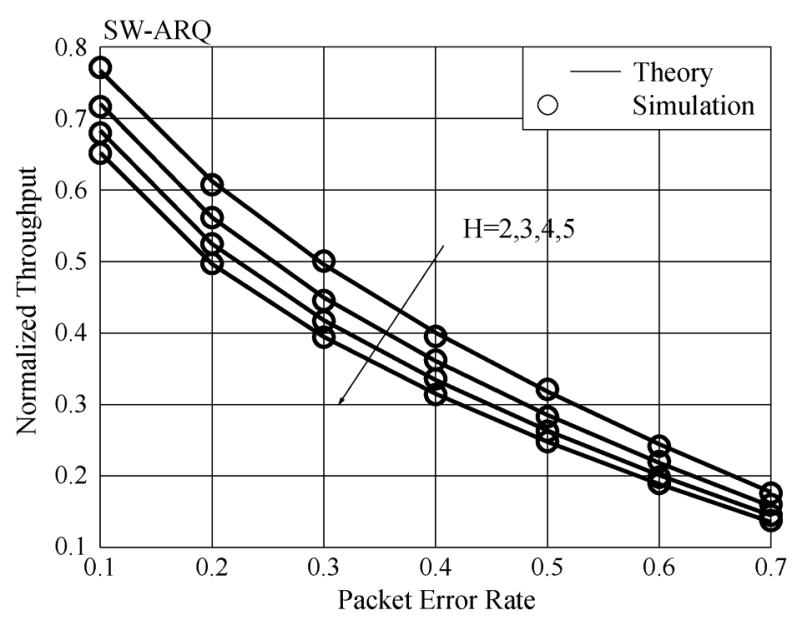

Fig. 9. Steady-state throughput versus packet error rate performance of the network coding node associated with $H=2,3,4,5$ incoming links.

coding node, when it has $H=2,3,4,5$ incoming links. The results of Fig. 9 show that at a given packet error rate $p_{e}$, the normalized throughput decreases when the coding node has more incoming links. This is because, explicitly, the chance of forming a new packet and forwarding it to buffer $b_{0}$ becomes smaller, as the new packet depends on correctly receiving more packets. Additionally, from Fig. 9 we observe that the difference of the normalized throughput corresponding to different number of incoming links becomes smaller as the packet error rate increases. 


\section{CONCLUSION}

In this paper, we have investigated the steady-state throughput of network coding nodes when the simple SW-ARQ transmission scheme is employed. Expressions for computing the steady-state throughput have been obtained by first considering a specific 2ISO network coding node and then extending it to the more general MISO network coding nodes. The steady-state throughput performance is investigated by both simulation and numerical approaches. It can be shown that the simulation results justify our analytical expressions derived. Furthermore, our performance results show that the throughput of a coding node decreases as the number of source nodes feeding packets into the coding node increases. This property implies that, in a network coding system, the coding nodes may form the bottlenecks for information delivery. The method presented in this paper is versatile in the sense that the steady-state throughput of a network coding system can be evaluated by following the similar steps of our method, provided that a corresponding transition probability matrix $\boldsymbol{P}$ can be obtained. Our future research in this area will consider extensions to the network coding systems using other types ARQ schemes, where every buffer may store a number of packets, and to the network coding systems with multiple coding nodes.

\section{ACKNOWLEDGMENT}

The authors would like to thank the anonymous reviewers for their fruitful suggestions.

\section{REFERENCES}

[1] R. Ahlswede, N. Cai, S. Y. R. Li, and R. W. Yeung, "Network information flow," IEEE Trans. Inf. Theory, vol. 46, no. 4, pp. 1204-1216, Jul. 2000.

[2] D. S. Lun, M. Medard, and M. Effros, "On coding for reliable communication over packet networks," in Proc. 42nd Annu. Allerton Conf. Commun., Control, Comput., Sept.-Oct. 2004, pp. 20-29.

[3] S. Y. R. Li, R. W. Yeung, and N. Cai, "Linear network coding," IEEE Trans. Inf. Theory, vol. 49, no. 2, pp. 371-381, Feb. 2003.

[4] D. S. Lun, M. Medard, R. Koetter, and M. Effros, "Further results on coding for reliable communication over packet networks," in Proc. IEEE Int. Symp. Inf. Theory, Sept. 4-9, 2005, pp. 1848-1852.

[5] S. Lin and D. J. Costello, Error Control Coding, 2nd ed. Upper Saddle River, NJ: Prentice-Hall, 2004.

[6] A. S. Tanenbaum, Computer Networks, 4th ed. Upper Saddle River, NJ: Prentice-Hall, 2003.

[7] Y. Hayashida, "Throughput analysis of tandem-type go-back-n ARQ scheme for satellite communications," IEEE Trans. Commun., vol. 41, no. 10, pp. 1517-1524, Oct. 1993.
[8] Y. Qin and L.-L. Yang, "Throughput analysis of stop-and-wait automatic repeat request scheme for network coding nodes," in Proc. IEEE VTC2010-Spring, Taipei, Taiwan, May 16-19, 2010, pp. 1-5.

[9] Y. Qin and L.-L. Yang, "Throughput analysis of general network coding nodes based on SW-ARQ transmission," in Proc. IEEE VTC2010-Fall, Ottawa, ON, Canada, Sep. 6-9, 2010, pp. 1-5.

[10] Y. Sagduyu and A. Ephremides, "On broadcast stability of queue-based dynamic network coding over erasure channels," IEEE Trans. Inf. Theory, vol. 55, no. 12, pp. 5463-5478, Dec. 2009.

[11] C. Fragouli, D. Lun, M. Medard, and P. Pakzad, "On feedback for network coding," in Proc. 41st Annu. Conf. Inf. Sci. Syst., Mar. 14-16, 2007, pp. 248-252.

[12] P. A. Chou, Y. Wu, and K. Jain, "Practical network coding," in Proc. 41st Annu. Allerton Conf. Commun., Control, Comput., 2003.

[13] J. K. Sundararajan, D. Shah, and M. Médard, "ARQ for network coding," in Proc. IEEE Int. Symp. Inf. Theory, Jul. 6-11, 2008, pp. 1651-1655.

[14] J. K. Sundararajan, D. Shah, and M. Médard, "Online network coding for optimal throughput and delay-the two-receiver case," Comput. Res. Repository, vol. abs/0806.4264, 2008.

[15] J. Barros, R. Costa, D. Munaretto, and J. Widmer, "Effective delay control in online network coding," in Proc. IEEE INFOCOM, Apr. 2009, pp. $208-216$.

[16] J. K. Sundararajan, P. Sadeghi, and M. Médard, "A feedback-based adaptive broadcast coding scheme for reducing in-order delivery delay," in Proc. Workshop Netw. Coding, Theory, Appl., Jun. 2009, pp. 1-6.

[17] D. Nguyen, T. Tran, T. Nguyen, and B. Bose, "Hybrid ARQ-random network coding for wireless media streaming," in Proc. 2nd Int. Conf. Commun. Electron., Jun. 4-6, 2008, pp. 115-120.

[18] Y. Chen, S. Kishore, and J. Li, "Wireless diversity through network coding," in Proc. IEEE WCNC, 2006, vol. 3, pp. 1681-1686.

[19] Y. Dallery, Z. Liu, and D. Towsley, "Equivalence, reversibility, symmetry and concavity properties in fork-join queuing networks with blocking," J. ACM, vol. 41, no. 5, pp. 903-942, 1994.

[20] Y. Dallery, Z. Liu, and D. Towsley, "Properties of fork/join queuing networks with blocking under various operating mechanisms," IEEE Trans. Robot. Autom., vol. 13, no. 4, pp. 503-518, Aug. 1997.

[21] R. Nelson and A. Tantawi, "Approximate analysis of fork/join synchronization in parallel queues," IEEE Trans. Comput., vol. 37, no. 6, pp. 739-743, Jun. 1988.

[22] C. Fragouli and E. Soljanin, "Information flow decomposition for network coding," IEEE Trans. Inf. Theory, vol. 52, no. 3, pp. 829-848, Mar. 2006

[23] A. Lebrecht and W. J. Knottenbelt, "Response time approximations in Fork-Join queues," in Proc. 23rd Annu. UKPEW, Jul. 2007 [Online]. Available: http://pubs.doc.ic.ac.uk/forkjoin

[24] S. M. Ross, Introduction to Probability and Statistics for Engineers and Scientists, 4th ed. New York: Academic, 2009.

[25] R. A. Horn, Matrix Analysis. Cambridge, U.K.: Cambridge Univ. Press, 1990

[26] D. P. Bertsekas and R. G. Gallager, Data Networks. Upper Saddle River, NJ: Prentice-Hall, 1992.

Yang Qin, photograph and biography not available at the time of publication.

Lie-Liang Yang photograph and biography not available at the time of publication. 